\title{
Two-bound core games and the nucleolus
}

Citation for published version (APA):

Gong, D., Dietzenbacher, B., \& Peters, H. (2021). Two-bound core games and the nucleolus. Maastricht University, Graduate School of Business and Economics. GSBE Research Memoranda No. 020 https://doi.org/10.26481/umagsb.2021020

Document status and date:

Published: 13/12/2021

DOI:

10.26481/umagsb.2021020

Document Version:

Publisher's PDF, also known as Version of record

\section{Please check the document version of this publication:}

- A submitted manuscript is the version of the article upon submission and before peer-review. There can be important differences between the submitted version and the official published version of record.

People interested in the research are advised to contact the author for the final version of the publication, or visit the DOI to the publisher's website.

- The final author version and the galley proof are versions of the publication after peer review.

- The final published version features the final layout of the paper including the volume, issue and page numbers.

Link to publication

\footnotetext{
General rights rights.

- You may freely distribute the URL identifying the publication in the public portal. please follow below link for the End User Agreement:

www.umlib.nl/taverne-license

Take down policy

If you believe that this document breaches copyright please contact us at:

repository@maastrichtuniversity.nl

providing details and we will investigate your claim.
}

Copyright and moral rights for the publications made accessible in the public portal are retained by the authors and/or other copyright owners and it is a condition of accessing publications that users recognise and abide by the legal requirements associated with these

- Users may download and print one copy of any publication from the public portal for the purpose of private study or research.

- You may not further distribute the material or use it for any profit-making activity or commercial gain

If the publication is distributed under the terms of Article $25 \mathrm{fa}$ of the Dutch Copyright Act, indicated by the "Taverne" license above, 


\section{Maastricht University}

Doudou Gong

Bas Dietzenbacher

Hans Peters

Two-bound core games and the nucleolus

$\mathrm{RM} / 21 / 020$

ISSN: 2666-8807

\section{GSBE}

Maastricht University School of Business and Economics

Graduate School of Business and Economics

P.O Box 616

NL- 6200 MD Maastricht

The Netherlands 


\title{
Two-bound core games and the nucleolus
}

\author{
Doudou Gong ${ }^{1,2 *} \quad$ Bas Dietzenbacher ${ }^{2 \dagger} \quad$ Hans Peters $^{2 \ddagger}$
}

December 7, 2021

\begin{abstract}
This paper introduces the new class of two-bound core games, where the core can be described by a lower bound and an upper bound on the payoffs of the players. Many classes of games turn out to be two-bound core games. We show that the core of each two-bound core game can be described equivalently by the pair of exact core bounds, and study to what extent the exact core bounds can be stretched while retaining the core description. We provide explicit expressions of the nucleolus for two-bound core games in terms of all pairs of bounds describing the core, using the Talmud rule for bankruptcy problems, and study to what extent these expressions are robust against game changes.
\end{abstract}

Keywords: two-bound core games, nucleolus, Talmud rule

JEL classification: C71

\section{Introduction}

In the theory of cooperative games (with transferable utility), players collaborate in coalitions to generate profits. Cooperative game theory analyzes how to allocate profits generated by the grand coalition among the players in a fair way, and provides several significant solution concepts.

A central solution concept is the core, which consists of all coalitionally stable preimputations, that is, no coalition will obtain more by deviating from cooperation in the grand coalition. Bondareva (1963) and Shapley (1967) showed that the core is nonempty if and only if the corresponding cooperative game is balanced. Another important solution concept is the nucleolus (cf. Schmeidler 1969), which lexicographically minimizes the excesses of coalitions. The nucleolus selects from the core in each balanced game.

\footnotetext{
*Corresponding author. E-mail: d.gong@maastrichtuniversity.nl.

$\dagger$ E-mail: b.dietzenbacher@maastrichtuniversity.nl.

‡E-mail: h.peters@maastrichtuniversity.nl.

${ }^{1}$ School of Mathematics and Statistics, Northwestern Polytechnical University, Xi'an, 710072, China.

${ }^{2}$ Department of Quantitative Economics, Maastricht University, Maastricht, 6200 MD, The Netherlands.
} 
Quant et al. (2005) studied the class of compromise stable games where the core coincides with the core cover (cf. Tijs and Lipperts 1982), and provided an explicit expression of the nucleolus for this class using the Talmud rule for bankruptcy problems. The core cover is the set of pre-imputations between a specific pair of bounds. In this paper, we generalize the approach of Quant et al. (2005) to all games where the core equals the set of pre-imputations between an arbitrary pair of bounds, which we call two-bound core games.

We show that the core of each two-bound core game can be described equivalently by the pair of exact core bounds (cf. Bondareva and Driessen 1994), which are defined by the minimum and maximum individual payoffs within the core. Inspired by Quant et al. (2005), we provide conditions to check whether a game is a two-bound core game, and describe the extreme points of the core for each such game. All balanced games with at most three players are two-bound core games, but this does not hold for more players.

We study to what extent the exact core bounds of a two-bound core game can be stretched while retaining the core description. It turns out that only three possible cases exist. In the first case, only the lower bounds are decreased for players who obtain their lower exact core bounds when all other players obtain their upper exact core bounds, while keeping all other bounds fixed. In the second case, only the upper bounds are increased for players who obtain their upper exact core bounds when all other players obtain their lower exact core bounds, while keeping all other bounds fixed. In the third case, both the lower bound is decreased and the upper bound is increased for only a single player who obtains the lower exact core bound when all other players obtain their upper exact core bounds and obtains the upper exact core bound when all other players obtain their lower exact core bounds.

In line with Quant et al. (2005), we provide an explicit expression of the nucleolus for two-bound core games in terms of the exact core bounds using the Talmud rule. In fact, the nucleolus of these games can be equivalently expressed by each pair of bounds describing the core. We study to what extent these expressions are robust against game changes.

The remainder of this paper is organized as follows. Section 2 introduces preliminary definitions and notation about cooperative games and bankruptcy problems. In Section 3 , we formally introduce two-bound core games. The nucleolus for two-bound core games is studied in Section 4 Finally, we conclude this paper with some remarks in Section 5.

\section{Preliminaries}

Let $N$ be a nonempty and finite set of players and let $2^{N}$ be the collection of all subsets of $N$. An order of $N$ is a bijection $\sigma:\{1, \ldots,|N|\} \rightarrow N$, where $|N|$ denotes the cardinality of $N$, and $\sigma(i)$ represents the player at position $i$. The set of all orders of $N$ is denoted by $\Pi(N)$. Denote by $\mathbb{R}_{+}$the set of all non-negative real numbers. 
Let $x, y \in \mathbb{R}^{N}$. We denote $x+y=\left(x_{i}+y_{i}\right)_{i \in N}, x-y=\left(x_{i}-y_{i}\right)_{i \in N}$, and $\lambda x=\left(\lambda x_{i}\right)_{i \in N}$ for all $\lambda \in \mathbb{R}$. Moreover, $x \geq y$ denotes $x_{i} \geq y_{i}$ for all $i \in N$, and $x>y$ denotes $x_{i}>y_{i}$ for all $i \in N$. The notations $\leq$ and $<$ are defined analogously. We denote

$$
[x, y]=\left\{z \in \mathbb{R}^{N} \mid x \leq z \leq y\right\}
$$

A cooperative game with transferable utility (a game, for short) is a pair $(N, v)$, where $v: 2^{N} \rightarrow \mathbb{R}$ is the characteristic function with $v(\emptyset)=0$, representing the worth $v(S)$ for each coalition $S \subseteq N$ when the players in $S$ cooperate. The set of all games with player set $N$ is denoted by $\Gamma^{N}$. For simplicity, we write $v \in \Gamma^{N}$ rather than $(N, v) \in \Gamma^{N}$.

Let $v \in \Gamma^{N}$. The pre-imputation set of $v$ is

$$
X(v)=\left\{x \in \mathbb{R}^{N} \mid \sum_{i \in N} x_{i}=v(N)\right\},
$$

the imputation set of $v$ is

$$
I(v)=\left\{x \in X(v) \mid \forall i \in N: x_{i} \geq v(\{i\})\right\},
$$

and the core of $v$ is

$$
C(v)=\left\{x \in X(v) \mid \forall S \subseteq N: \sum_{i \in S} x_{i} \geq v(S)\right\} .
$$

Note that $C(v) \subseteq I(v) \subseteq X(v)$, and $C(\lambda v+a)=\lambda C(v)+a$ for all $\lambda \in \mathbb{R}_{+}$and $a \in \mathbb{R}^{N}$, where $\lambda v+a \in \Gamma^{N}$ is defined by $(\lambda v+a)(S)=\lambda v(S)+\sum_{i \in S} a_{i}$ for all $S \subseteq N$.

Bondareva (1963) and Shapley (1967) showed that a game $v \in \Gamma^{N}$ is balanced if and only if $C(v) \neq \emptyset$. The set of all balanced games with player set $N$ is denoted by $\Gamma_{b}^{N}$. A game $v \in \Gamma^{N}$ is convex (cf. Shapley 1971) if $v(S)+v(T) \leq v(S \cup T)+v(S \cap T)$ for all $S, T \subseteq N$. The set of all convex games with player set $N$ is denoted by $\Gamma_{c}^{N}$. It is known that $\Gamma_{c}^{N} \subseteq \Gamma_{b}^{N} \subseteq \Gamma^{N}$.

A value $\varphi$ on a domain of games assigns to each game $v$ in this domain a pre-imputation $\varphi(v) \in X(v)$. The nucleolus (cf. Schmeidler 1969) is the value $\eta$ that assigns to each game $v \in \Gamma^{N}$ with $I(v) \neq \emptyset$ the unique imputation $x \in I(v)$ satisfying $\theta(x) \preccurlyeq \theta(y)$ for all $y \in I(v)$, where $\theta(x) \in \mathbb{R}^{2^{|N|}-2}$ is the vector of excesses $v(S)-\sum_{i \in S} x_{i}$ for all $S \in 2^{N} \backslash\{N, \emptyset\}$ arranged in non-increasing order, i.e., $\theta_{k}(x) \geq \theta_{\ell}(x)$ for all $1 \leq k<\ell \leq 2^{|N|}-2$, and $\theta(x) \preccurlyeq \theta(y)$ if there exists $1 \leq t \leq 2^{|N|}-2$ such that $\theta_{t}(x)<\theta_{t}(y)$ and $\theta_{k}(x)=\theta_{k}(y)$ for all $1 \leq k<t$, or $\theta(x)=\theta(y)$. It is easy to see that $\eta(v) \in C(v)$ for all $v \in \Gamma_{b}^{N}$, and $\eta(\lambda v+a)=\lambda \eta(v)+a$ for all $\lambda \in \mathbb{R}_{+}$and $a \in \mathbb{R}^{N}$. 
A bankruptcy problem is a triple $(N, E, c)$, where $E \in \mathbb{R}_{+}$is the estate to be divided and $c \in \mathbb{R}_{+}^{N}$ is the vector of claims satisfying $\sum_{i \in N} c_{i} \geq E$. The set of all bankruptcy problems with player set $N$ is denoted by $\mathcal{B}^{N}$. For simplicity, we write $(E, c) \in \mathcal{B}^{N}$ rather than $(N, E, c) \in \mathcal{B}^{N}$.

A bankruptcy rule $f: \mathcal{B}^{N} \rightarrow \mathbb{R}_{+}^{N}$ assigns to each bankruptcy problem $(E, c) \in \mathcal{B}^{N}$ a payoff vector $f(E, c) \in \mathbb{R}_{+}^{N}$ such that $\sum_{i \in N} f_{i}(E, c)=E$ and $f_{i}(E, c) \leq c_{i}$ for all $i \in N$. A bankruptcy rule $f$ is self-dual (cf. Aumann and Maschler 1985) if for all $(E, c) \in \mathcal{B}^{N}$,

$$
f(E, c)=c-f\left(\sum_{i \in N} c_{i}-E, c\right) .
$$

A bankruptcy rule $f$ is invariant under claims truncation if for all $(E, c) \in \mathcal{B}^{N}$,

$$
f(E, c)=f\left(E,\left(\min \left\{c_{i}, E\right\}\right)_{i \in N}\right) .
$$

The Talmud (TAL) rule assigns to each bankruptcy problem $(E, c) \in \mathcal{B}^{N}$ and each player $i \in N$,

$$
f_{i}^{T A L}(E, c)=\left\{\begin{array}{l}
\min \left\{c_{i} / 2, \lambda\right\}, \text { if } \sum_{i \in N} c_{i} \geq 2 E, \\
\max \left\{c_{i} / 2, c_{i}-\lambda\right\}, \text { if } \sum_{i \in N} c_{i}<2 E,
\end{array}\right.
$$

where $\lambda \in \mathbb{R}$ is such that $\sum_{i \in N} f_{i}^{T A L}(E, c)=E$. Aumann and Maschler (1985) showed that the Talmud rule is self-dual and invariant under claims truncation.

The bankruptcy game (cf. O'Neill 1982) $v_{E, c} \in \Gamma^{N}$ associated to bankruptcy problem $(E, c) \in \mathcal{B}^{N}$ assigns to each coalition $S \subseteq N$ the residual estate after all other claims have been satisfied, i.e.,

$$
v_{E, c}(S)=\max \left\{0, E-\sum_{i \in N \backslash S} c_{i}\right\} .
$$

Curiel et al. (1987) showed that bankruptcy games are convex games. Aumann and Maschler (1985) showed that for each bankruptcy problem, the payoff vector assigned by the Talmud rule coincides with the nucleolus of the corresponding bankruptcy game.

\section{Two-bound core games}

In this section, we introduce two-bound core games, where the core equals the set of preimputations between a lower bound and an upper bound. Let $v \in \Gamma^{N}$. Given $l, u \in \mathbb{R}^{N}$, the $l, u$-efficient set of $v$

$$
[l, u] \cap X(v)
$$

consists of all pre-imputations between lower bound $l$ and upper bound $u$, i.e., it is the intersection of the pre-imputation set and the $|N|$-dimensional hypercube restricted by $l$ and 
$u$, so it is a convex set. If this set is nonempty, then its extreme points can be described as follows. Similar to Quant et al. (2005), we define $m^{l, u, \sigma}(v) \in \mathbb{R}^{N}$ for all $\sigma \in \Pi(N)$ and all $k \in\{1, \ldots,|N|\}$ by

$$
m_{\sigma(k)}^{l, u, \sigma}(v)=\left\{\begin{array}{l}
u_{\sigma(k)}, \text { if } \sum_{j=1}^{k} u_{\sigma(j)}+\sum_{j=k+1}^{|N|} l_{\sigma(j)} \leq v(N), \\
l_{\sigma(k)}, \text { if } \sum_{j=1}^{k-1} u_{\sigma(j)}+\sum_{j=k}^{|N|} l_{\sigma(j)} \geq v(N) \\
v(N)-\sum_{j=1}^{k-1} u_{\sigma(j)}-\sum_{j=k+1}^{|N|} l_{\sigma(j)}, \text { otherwise. }
\end{array}\right.
$$

Thus, $m^{l, u, \sigma}(v)$ assigns to the first players in $\sigma$ their upper bound payoffs in such a way that the last players in $\sigma$ are assigned their lower bound payoffs. The pivot player of $m^{l, u, \sigma}(v)$ is the first player in $\sigma$ who is not assigned the upper bound payoff. If all the players receive their upper bound payoffs, then the last player is the pivot player of $m^{l, u, \sigma}(v)$. These definitions are straightforward generalizations of concepts in Quant et al. (2005) to arbitrary lower and upper bounds, which can be used to describe the $l, u$-efficient set.

\section{Lemma 1}

Let $v \in \Gamma^{N}$ and let $l, u \in \mathbb{R}^{N}$ be such that $[l, u] \cap X(v) \neq \emptyset$. Then

$$
[l, u] \cap X(v)=\operatorname{conv}\left\{m^{l, u, \sigma}(v) \mid \sigma \in \Pi(N)\right\} .
$$

Proof. In view of $m^{l, u, \sigma}(v) \in[l, u] \cap X(v)$ for all $\sigma \in \Pi(N)$, together with the convexity of $[l, u] \cap X(v)$ and $\operatorname{conv}\left\{m^{l, u, \sigma}(v) \mid \sigma \in \Pi(N)\right\}$, we have

$$
\operatorname{conv}\left\{m^{l, u, \sigma}(v) \mid \sigma \in \Pi(N)\right\} \subseteq[l, u] \cap X(v) .
$$

Let $x \in \mathbb{R}^{N}$ be an arbitrary extreme point of $[l, u] \cap X(v)$, i.e., for each $0<\lambda<1$ and all $y, z \in[l, u] \cap X(v), \lambda y+(1-\lambda) z=x$ implies that $x=y=z$. We claim that there exists at most one player $i \in N$ such that $l_{i}<x_{i}<u_{i}$ and $\left[x_{j}=l_{j}\right.$ or $x_{j}=u_{j}$ for all $\left.j \in N \backslash\{i\}\right]$. Assume, to the contrary, that there exist $i, j \in N$ with $i \neq j$ such that $l_{i}<x_{i}<u_{i}$ and $l_{j}<x_{j}<u_{j}$. Let $0<\varepsilon<\min \left\{x_{i}-l_{i}, u_{i}-x_{i}, x_{j}-l_{j}, u_{j}-x_{j}\right\}$, let $x^{\prime}$ be defined by $x_{i}^{\prime}=x_{i}+\varepsilon$, $x_{j}^{\prime}=x_{j}-\varepsilon$ and $x_{k}^{\prime}=x_{k}$ for all $k \in N \backslash\{i, j\}$, and let $x^{\prime \prime}$ be defined by $x_{i}^{\prime \prime}=x_{i}-\varepsilon, x_{j}^{\prime \prime}=x_{j}+\varepsilon$ and $x_{k}^{\prime \prime}=x_{k}$ for all $k \in N \backslash\{i, j\}$. Then $x^{\prime}, x^{\prime \prime} \in[l, u] \cap X(v)$ and $x=\frac{1}{2} x^{\prime}+\frac{1}{2} x^{\prime \prime}$, which contradicts the fact that $x$ is an extreme point of $[l, u] \cap X(v)$.

If $x_{i}=l_{i}$ or $x_{i}=u_{i}$ for all $i \in N$, then it holds that $x=m^{l, u, \sigma}(v)$ for all $\sigma \in \Pi(N)$ such that $x_{\sigma(k)}=u_{\sigma(k)}$ if and only if $k \leq\left|\left\{i \in N \mid x_{i}=u_{i}\right\}\right|$. If there exists $i \in N$ such that $l_{i}<x_{i}<u_{i}$ and $\left[x_{j}=l_{j}\right.$ or $x_{j}=u_{j}$ for all $\left.j \in N \backslash\{i\}\right]$, then it holds that $x=m^{l, u, \sigma}(v)$ for all $\sigma \in \Pi(N)$ such that $\left[x_{\sigma(k)}=u_{\sigma(k)}\right.$ if and only if $\left.k \leq\left|\left\{j \in N \mid x_{j}=u_{j}\right\}\right|\right]$ and $\sigma(\mid\{j \in N \mid$ $\left.\left.x_{j}=u_{j}\right\} \mid+1\right)=i$. Again with the convexity of $[l, u] \cap X(v)$ and $\operatorname{conv}\left\{m^{l, u, \sigma}(v) \mid \sigma \in \Pi(N)\right\}$, we have $[l, u] \cap X(v) \subseteq \operatorname{conv}\left\{m^{l, u, \sigma}(v) \mid \sigma \in \Pi(N)\right\}$. 
The $l, u$-efficient set and the core are both convex subsets of the pre-imputation set. We are interested in $l, u$-efficient sets that contain the core. Many well-known sets are of this type, such as the imputation set and the core cover (cf. Tijs and Lipperts 1982).

\section{Example 1}

Let $v \in \Gamma^{N}$. Define $l, u \in \mathbb{R}^{N}$ by

$$
l_{i}=v(\{i\}) \text { and } u_{i}=v(N)-\sum_{j \in N \backslash\{i\}} v(\{j\})
$$

for all $i \in N$. Then $[l, u] \cap X(v)=I(v)$, so the $l$,u-efficient set contains the core.

\section{Example 2}

Let $v \in \Gamma^{N}$. Define $l, u \in \mathbb{R}^{N}$ by

$$
l_{i}=v(\{i\}) \text { and } u_{i}=v(N)-v(N \backslash\{i\})
$$

for all $i \in N$. Then $C(v) \subseteq[l, u] \cap X(v)$, i.e., the $l$, $u$-efficient set contains the core.

\section{Example 3}

Let $v \in \Gamma^{N}$. Define $l, u \in \mathbb{R}^{N}$ by

$$
l_{i}=\max _{S \in 2^{N}: i \in S}\left\{v(S)-\sum_{j \in S \backslash\{i\}}(v(N)-v(N \backslash\{j\}))\right\} \text { and } u_{i}=v(N)-v(N \backslash\{i\})
$$

for all $i \in N$. Then $[l, u] \cap X(v)$ defines the core cover (cf. Tijs and Lipperts 1982), which contains the core. Quant et al. (2005) defined compromise stable games as games where the core cover coincides with the core.

To check whether a core-containing $l, u$-efficient set coincides with the core, we only need to verify a specific inequality for each nonempty coalition.

\section{Theorem 1}

Let $v \in \Gamma_{b}^{N}$ and let $l, u \in \mathbb{R}^{N}$ be such that $C(v) \subseteq[l, u]$. Then $C(v)=[l, u] \cap X(v)$ if and only if for each $S \in 2^{N} \backslash\{\emptyset\}$,

$$
v(S) \leq \max \left\{\sum_{i \in S} l_{i}, v(N)-\sum_{i \in N \backslash S} u_{i}\right\} .
$$

Theorem 1, the proof of which is in the Appendix, generalizes the work of Quant et al. (2005), where this result was proven for the specific pair of bounds in Example 3 If the $l, u$-efficient set does not contain the core, then expression (1) may hold even when the core does not coincide with the $l, u$-efficient set. This is shown by the following example. 


\section{Example 4}

Let $N=\{1,2\}$ and let $v \in \Gamma^{N}$ be given by $v(\{1\})=1, v(\{2\})=2$ and $v(N)=4$. Define $l, u \in \mathbb{R}^{N}$ by $l_{1}=u_{1}=\frac{3}{2}$ and $l_{2}=u_{2}=\frac{5}{2}$. It is easy to verify that expression 11 holds for each nonempty coalition. However, $C(v)=\left\{x \in \mathbb{R}^{N} \mid x_{1}+x_{2}=4, x_{1} \geq 1, x_{2} \geq 2\right\}$ and $[l, u] \cap X(v)=\left\{\left(\frac{3}{2}, \frac{5}{2}\right)\right\}$. Clearly, $C(v) \neq[l, u] \cap X(v)$.

We focus on games where the core coincides with some $l, u$-efficient set. These games are called two-bound core games.

\section{Definition 1}

A game $v \in \Gamma_{b}^{N}$ is a two-bound core game if there exist $l, u \in \mathbb{R}^{N}$ such that

$$
C(v)=[l, u] \cap X(v) .
$$

The set of all two-bound core games with player set $N$ is denoted by $\Gamma_{t}^{N}$. It is worthwhile mentioning that many classical games are two-bound core games. For example, additive games, unanimity games, bankruptcy games (cf. O’Neill 1982), 1-convex games (cf. Driessen 1986), big boss games (cf. Muto et al. 1988), clan games (cf. Potters et al. 1989), compromise stable games (cf. Quant et al. 2005) and reasonable stable games (cf. Dietzenbacher 2018).

It turns out that the core of each two-bound core game can be described by the following specific pair of bounds. Let $v \in \Gamma_{b}^{N}$. The lower exact core bound is defined by

$$
l_{i}^{*}(v)=\min _{x \in C(v)} x_{i} \quad \text { for all } i \in N .
$$

The upper exact core bound is defined by

$$
u_{i}^{*}(v)=\max _{x \in C(v)} x_{i} \quad \text { for all } i \in N
$$

The lower and upper exact core bounds were also studied by Bondareva and Driessen (1994).

\section{Lemma 2}

A game $v \in \Gamma_{b}^{N}$ is a two-bound core game if and only if $C(v)=\left[l^{*}(v), u^{*}(v)\right] \cap X(v)$.

Proof. The if-part follows directly from the definition of two-bound core games. For the onlyif part, assume that $C(v)=[l, u] \cap X(v)$ for some $l, u \in \mathbb{R}^{N}$. Then $l_{i} \leq l_{i}^{*}(v)$ and $u_{i} \geq u_{i}^{*}(v)$ for all $i \in N$, so $\left[l^{*}(v), u^{*}(v)\right] \subseteq[l, u]$. Together with $C(v) \subseteq\left[l^{*}(v), u^{*}(v)\right] \cap X(v)$, it follows that $C(v) \subseteq\left[l^{*}(v), u^{*}(v)\right] \cap X(v) \subseteq[l, u] \cap X(v)=C(v)$. Hence, $C(v)=\left[l^{*}(v), u^{*}(v)\right] \cap$ $X(v)$.

All balanced games with at most three players are two-bound core games, but this does not hold for more players. 


\section{Proposition 1}

$\Gamma_{t}^{N}=\Gamma_{b}^{N}$ if and only if $|N| \leq 3$.

Proof. Let $v \in \Gamma_{b}^{N}$ with $|N|=2$. Then it can be seen directly that $v \in \Gamma_{t}^{N}$ since $l_{i}^{*}(v)=v(\{i\})$ and $u_{i}^{*}(v)=v(N)-v(N \backslash\{i\})$ for all $i \in N$, which implies that $v(S) \leq$ $\max \left\{\sum_{i \in S} l_{i}^{*}(v), v(N)-\sum_{i \in N \backslash S} u_{i}^{*}(v)\right\}$ for all $S \in 2^{N} \backslash\{\emptyset\}$, so Theorem 1 applies.

Let $v \in \Gamma_{b}^{N}$ with $|N|=3$. For all $i \in N$,

$$
v(\{i\}) \leq l_{i}^{*}(v) \leq \max \left\{l_{i}^{*}(v), v(N)-\sum_{j \in N \backslash\{i\}} u_{j}^{*}(v)\right\} .
$$

For all $S \in 2^{N}$ with $|S|=2$,

$$
v(S) \leq v(N)-\sum_{i \in N \backslash S} u_{i}^{*}(v) \leq \max \left\{\sum_{i \in S} l_{i}^{*}(v), v(N)-\sum_{i \in N \backslash S} u_{i}^{*}(v)\right\} .
$$

Hence, $v \in \Gamma_{t}^{N}$ by Theorem 1 .

Let $v \in \Gamma_{b}^{N}$ with $|N|>3$ be defined by $v(N)=3, v(\{i, j\})=1$ for distinct $i, j \in N$ and $v(S)=0$ otherwise. Then $l_{k}^{*}(v)=0$ for all $k \in N, u_{i}^{*}(v)=u_{j}^{*}(v)=3$, and $u_{k}^{*}(v)=2$ for all $k \in N \backslash\{i, j\}$. This implies that

$$
v(\{i, j\})=1>0+0=l_{i}^{*}(v)+l_{j}^{*}(v)
$$

and

$$
v(\{i, j\})=1>3-2(|N|-2)=v(N)-\sum_{k \in N \backslash\{i, j\}} u_{k}^{*}(v) .
$$

Hence, $v \notin \Gamma_{t}^{N}$ by Theorem 1 and Lemma 2

In what follows next, we study to what extent the exact core bounds of a two-bound core game can be stretched while retaining the core description. It turns out that the exact core bounds can be stretched in only three different ways.

\section{Proposition 2}

Let $v \in \Gamma_{t}^{N}$. If there exist $l, u \in \mathbb{R}^{N}$ with $[l, u] \neq\left[l^{*}(v), u^{*}(v)\right]$ such that $C(v)=[l, u] \cap X(v)$, then exactly one of the following cases holds:

(i) $l \leq l^{*}(v)$ and $u=u^{*}(v)$,

(ii) $l=l^{*}(v)$ and $u \geq u^{*}(v)$,

(iii) there exists $i \in N$ such that $l_{i}<l_{i}^{*}(v), u_{i}>u_{i}^{*}(v)$, and $l_{j}=l_{j}^{*}(v)$ and $u_{j}=u_{j}^{*}(v)$ for all $j \in N \backslash\{i\}$. 
Proof. In view of $l \leq l^{*}(v)$ and $u \geq u^{*}(v)$, it suffices to prove that if $l \neq l^{*}(v)$ and $u \neq u^{*}(v)$, then case (iii) arises. Assume to the contrary that there exist $i, j \in N$ with $i \neq j$ such that $l_{i}<l_{i}^{*}(v)$ and $u_{j}>u_{j}^{*}(v)$. Let $x \in C(v)$. Define $x^{\prime}$ by $x_{i}^{\prime}=x_{i}-\varepsilon, x_{j}^{\prime}=x_{j}+\varepsilon$ and $x_{k}^{\prime}=x_{k}$ for all $k \in N \backslash\{i, j\}$, where $\varepsilon=\min \left\{x_{i}-l_{i}, u_{j}-x_{j}\right\} \geq \min \left\{l_{i}^{*}(v)-l_{i}, u_{j}-u_{j}^{*}(v)\right\}>0$. Then $x^{\prime} \in[l, u] \cap X(v)$, but $x^{\prime} \notin C(v)$ in view of $x_{i}^{\prime}=l_{i}<l_{i}^{*}(v)$ or $x_{j}^{\prime}=u_{j}>u_{i}^{*}(v)$. So, $C(v) \neq[l, u] \cap X(v)$, which is a contradiction.

Moreover, we show that the first case in Proposition 2 arises only if the players whose lower bounds are decreased obtain their lower exact core bounds when all other players obtain their upper exact core bounds. The second case in Proposition 2 arises only if the players whose upper bounds are increased obtain their upper exact core bounds when all other players obtain their lower exact core bounds. The third case in Proposition 2 arises only if the player whose exact core bounds are stretched obtains the lower exact core bound when all other players obtain their upper exact core bounds and obtains the upper exact core bound when all other players obtain their lower exact core bounds.

\section{Theorem 2}

Let $v \in \Gamma_{t}^{N}$ and let $l, u \in \mathbb{R}^{N}$. Then the following statements hold:

(i) If $l \leq l^{*}(v)$ and $u=u^{*}(v)$, then $C(v)=[l, u] \cap X(v)$ if and only if

$$
v(N)=l_{i}^{*}(v)+\sum_{j \in N \backslash\{i\}} u_{j}^{*}(v) \text { for all } i \in N \text { with } l_{i}<l_{i}^{*}(v) .
$$

(ii) If $l=l^{*}(v)$ and $u \geq u^{*}(v)$, then $C(v)=[l, u] \cap X(v)$ if and only if

$$
v(N)=u_{i}^{*}(v)+\sum_{j \in N \backslash\{i\}} l_{j}^{*}(v) \text { for all } i \in N \text { with } u_{i}>u_{i}^{*}(v) .
$$

(iii) If there exists $i \in N$ such that $l_{i}<l_{i}^{*}(v), u_{i}>u_{i}^{*}(v)$, and $l_{j}=l_{j}^{*}(v)$ and $u_{j}=u_{j}^{*}(v)$ for all $j \in N \backslash\{i\}$, then $C(v)=[l, u] \cap X(v)$ if and only if

$$
u_{i}^{*}(v)+\sum_{j \in N \backslash\{i\}} l_{j}^{*}(v)=v(N)=l_{i}^{*}(v)+\sum_{j \in N \backslash\{i\}} u_{j}^{*}(v) .
$$

Proof. (2) For the only-if part, assume that $C(v)=[l, u] \cap X(v)$, where $l \leq l^{*}(v)$ and $u=$ $u^{*}(v)$. We show that $v(N)=l_{i}^{*}(v)+\sum_{j \in N \backslash\{i\}} u_{j}^{*}(v)$ for all $i \in N$ with $l_{i}<l_{i}^{*}(v)$. Assume, to the contrary, that there exists $i \in N$ with $l_{i}<l_{i}^{*}(v)$ such that $v(N) \neq l_{i}^{*}(v)+\sum_{j \in N \backslash\{i\}} u_{j}^{*}(v)$. Let $x \in C(v)$ be such that $x_{i}=l_{i}^{*}(v)$. Then we have

$$
v(N)=x_{i}+\sum_{j \in N \backslash\{i\}} x_{j}<l_{i}^{*}(v)+\sum_{j \in N \backslash\{i\}} u_{j}^{*}(v) .
$$


It follows that there exists $j \in N \backslash\{i\}$ such that $x_{j}<u_{j}^{*}(v)$. Define $x^{\prime}$ by $x_{i}^{\prime}=x_{i}-\varepsilon$, $x_{j}^{\prime}=x_{j}+\varepsilon$ and $x_{k}^{\prime}=x_{k}$ for all $k \in N \backslash\{i, j\}$, where $0<\varepsilon<\min \left\{x_{i}-l_{i}, u_{j}^{*}(v)-x_{j}\right\}$. Then $x^{\prime} \in[l, u] \cap X(v)$, but $x^{\prime} \notin C(v)$ in view of $x_{i}^{\prime}<x_{i}=l_{i}^{*}(v)$. So, $C(v) \neq[l, u] \cap X(v)$, which is a contradiction.

For the if-part, assume that $l \leq l^{*}(v)$ and $u=u^{*}(v)$ such that $v(N)=l_{i}^{*}(v)+$ $\sum_{j \in N \backslash\{i\}} u_{j}^{*}(v)$ for all $i \in N$ with $l_{i}<l_{i}^{*}(v)$. We show that $C(v)=[l, u] \cap X(v)$. In view of $C(v)=\left[l^{*}(v), u^{*}(v)\right] \cap X(v) \subseteq[l, u] \cap X(v)$, we only need to prove that $[l, u] \cap X(v) \subseteq$ $\left[l^{*}(v), u^{*}(v)\right] \cap X(v)$. Let $x \in[l, u] \cap X(v)$. Then $x_{i} \geq l_{i}=l_{i}^{*}(v)$ for all $i \in N$ with $l_{i}=l_{i}^{*}(v)$. For all $i \in N$ with $l_{i}<l_{i}^{*}(v)$,

$$
x_{i}=v(N)-\sum_{j \in N \backslash\{i\}} x_{j} \geq v(N)-\sum_{j \in N \backslash\{i\}} u_{j}=v(N)-\sum_{j \in N \backslash\{i\}} u_{j}^{*}(v)=l_{i}^{*}(v) .
$$

Together with $x \leq u=u^{*}(v)$, we obtain that $x \in\left[l^{*}(v), u^{*}(v)\right] \cap X(v)$. Hence, $[l, u] \cap X(v) \subseteq$ $\left[l^{*}(v), u^{*}(v)\right] \cap X(v)$.

(ii) The proof is analogous to the proof of (i).

(iii) For the only-if part, assume that $C(v)=[l, u] \cap X(v)$, where $l_{i}<l_{i}^{*}(v), u_{i}>u_{i}^{*}(v)$, and $l_{j}=l_{j}^{*}(v)$ and $u_{j}=u_{j}^{*}(v)$ for all $j \in N \backslash\{i\}$. We show that expression 22 holds. Assume that $v(N) \neq u_{i}^{*}(v)+\sum_{j \in N \backslash\{i\}} l_{j}^{*}(v)$ or $v(N) \neq l_{i}^{*}(v)+\sum_{j \in N \backslash\{i\}} u_{j}^{*}(v)$. Then, analogous to the proofs of (i) and (ii), it follows that $C(v) \neq[l, u] \cap X(v)$, which is a contradiction.

For the if-part, assume that there exists $i \in N$ such that $l_{i}<l_{i}^{*}(v), u_{i}>u_{i}^{*}(v), l_{j}=l_{j}^{*}(v)$ and $u_{j}=u_{j}^{*}(v)$ for all $j \in N \backslash\{i\}$, and expression $(2 p$ holds. We show that $C(v)=$ $[l, u] \cap X(v)$. In view of $C(v)=\left[l^{*}(v), u^{*}(v)\right] \cap X(v) \subseteq[l, u] \cap X(v)$, we only need to prove that $[l, u] \cap X(v) \subseteq\left[l^{*}(v), u^{*}(v)\right] \cap X(v)$. Let $x \in[l, u] \cap X(v)$. Then

$$
x_{i}=v(N)-\sum_{j \in N \backslash\{i\}} x_{j} \geq v(N)-\sum_{j \in N \backslash\{i\}} u_{j}=v(N)-\sum_{j \in N \backslash\{i\}} u_{j}^{*}(v)=l_{i}^{*}(v)
$$

and $x_{j} \geq l_{j}=l_{j}^{*}(v)$ for all $j \in N \backslash\{i\}$, so $x \geq l^{*}(v)$. Similarly,

$$
x_{i}=v(N)-\sum_{j \in N \backslash\{i\}} x_{j} \leq v(N)-\sum_{j \in N \backslash\{i\}} l_{j}=v(N)-\sum_{j \in N \backslash\{i\}} l_{j}^{*}(v)=u_{i}^{*}(v)
$$

and $x_{j} \leq u_{j}=u_{j}^{*}(v)$ for all $j \in N \backslash\{i\}$, so $x \leq u^{*}(v)$. It follows that $x \in\left[l^{*}(v), u^{*}(v)\right] \cap X(v)$. Hence, $[l, u] \cap X(v) \subseteq\left[l^{*}(v), u^{*}(v)\right] \cap X(v)$.

Proposition 2 and Theorem 2 directly imply the following result, which shows exactly under which condition two-bound core games can be described by different pairs of bounds. 


\section{Corollary 1}

Let $v \in \Gamma_{t}^{N}$. Then there exist $l, u \in \mathbb{R}^{N}$ with $[l, u] \neq\left[l^{*}(v), u^{*}(v)\right]$ such that $C(v)=[l, u] \cap$ $X(v)$ if and only if there exists $i \in N$ such that $v(N)=l_{i}^{*}(v)+\sum_{j \in N \backslash\{i\}} u_{j}^{*}(v)$ or $v(N)=$ $u_{i}^{*}(v)+\sum_{j \in N \backslash\{i\}} l_{j}^{*}(v)$.

\section{The nucleolus}

In this section, we consider the nucleolus of two-bound core games. Quant et al. (2005) provided an explicit expression of the nucleolus for compromise stable games in terms of the pair of bounds in Example 3 using the Talmud rule for bankruptcy problems. On the one hand, we extend their approach by providing an explicit expression of the nucleolus for all two-bound core games in terms of the exact core bounds. On the other hand, we show that the nucleolus can be equivalently expressed by each pair of bounds describing the core.

\section{Lemma 3}

Let $v \in \Gamma_{t}^{N}$. Then

$$
\begin{aligned}
\eta(v) & =l^{*}(v)+f^{T A L}\left(v(N)-\sum_{i \in N} l_{i}^{*}(v), u^{*}(v)-l^{*}(v)\right) \\
& =u^{*}(v)-f^{T A L}\left(\sum_{i \in N} u_{i}^{*}(v)-v(N), u^{*}(v)-l^{*}(v)\right) .
\end{aligned}
$$

The proof of Lemma 3, which is in the Appendix, is similar to the proof of Theorem 4.2 of Quant et al. (2005). However, as the following example shows, the expression obtained by Quant et al. (2005) in terms of the pair of bounds in Example 3 is not valid for all two-bound core games.

\section{Example 5}

Let $v \in \Gamma_{t}^{N}$ with $N=\{1, \ldots, n\}$ and $n \geq 4$ be defined by $v(N)=v(\{1,2\})=v(\{1,3\})=1$ and $v(S)=0$ otherwise. Then $l^{*}(v)=u^{*}(v)=(1,0, \ldots, 0)$ and $C(v)=\{(1,0, \ldots, 0)\}$, so

$$
\eta(v)=(1,0, \ldots, 0)+f^{T A L}(0,(0, \ldots, 0))=(1,0, \ldots, 0) .
$$

However, $v$ is not a compromise stable game, and $\eta(v)$ cannot be expressed using the lower bound $l=(0, \ldots, 0)$ and the upper bound $u=(1, \ldots, 1)$ from Example 3 in view of

$$
\eta(v) \neq(0, \ldots, 0)+f^{T A L}(1,(1, \ldots, 1))=\left(\frac{1}{n}, \ldots, \frac{1}{n}\right) .
$$

More generally, the nucleolus of two-bound core games can be equivalently expressed in terms of each pair of bounds describing the core. 


\section{Theorem 3}

Let $v \in \Gamma_{t}^{N}$. Then

$$
\eta(v)=l+f^{T A L}\left(v(N)-\sum_{i \in N} l_{i}, u-l\right)=u-f^{T A L}\left(\sum_{i \in N} u_{i}-v(N), u-l\right)
$$

for all $l, u \in \mathbb{R}^{N}$ such that $C(v)=[l, u] \cap X(v)$.

Proof. Let $l, u \in \mathbb{R}^{N}$ be such that $C(v)=[l, u] \cap X(v)$. If $[l, u]=\left[l^{*}(v), u^{*}(v)\right]$, then the statement follows directly from Lemma 3 . Suppose that $[l, u] \neq\left[l^{*}(v), u^{*}(v)\right]$. Then, by Proposition 2, exactly one of the following cases holds.

(ii) $l \leq l^{*}(v)$ and $u=u^{*}(v)$. By Theorem 2, $v(N)=l_{i}^{*}(v)+\sum_{j \in N \backslash\{i\}} u_{j}^{*}(v)$ for all $i \in N$ with $l_{i}<l_{i}^{*}(v)$. This implies that $\sum_{j \in N} u_{j}^{*}(v)-v(N)=u_{i}^{*}(v)-l_{i}^{*}(v)<u_{i}-l_{i}$ for all $i \in N$ with $l_{i}<l_{i}^{*}(v)$. Applying Lemma 3 invariance under claims truncation, and self-duality,

$$
\begin{aligned}
\eta(v) & =u^{*}(v)-f^{T A L}\left(\sum_{i \in N} u_{i}^{*}(v)-v(N), u^{*}(v)-l^{*}(v)\right) \\
& =u-f^{T A L}\left(\sum_{i \in N} u_{i}-v(N), u-l^{*}(v)\right) \\
& =u-f^{T A L}\left(\sum_{i \in N} u_{i}-v(N), u-l\right) \\
& =l+f^{T A L}\left(v(N)-\sum_{i \in N} l_{i}, u-l\right) .
\end{aligned}
$$

(ii) $l=l^{*}(v)$ and $u \geq u^{*}(v)$. By Theorem 2, $v(N)=u_{i}^{*}(v)+\sum_{j \in N \backslash\{i\}} l_{j}^{*}(v)$ for all $i \in N$ with $u_{i}>u_{i}^{*}(v)$. This implies that $v(N)-\sum_{j \in N} l_{j}^{*}(v)=u_{i}^{*}(v)-l_{i}^{*}(v)<u_{i}-l_{i}$ for all $i \in N$ with $u_{i}>u_{i}^{*}(v)$. Applying Lemma 3 , invariance under claims truncation, and self-duality,

$$
\begin{aligned}
\eta(v) & =l^{*}(v)+f^{T A L}\left(v(N)-\sum_{i \in N} l_{i}^{*}(v), u^{*}(v)-l^{*}(v)\right) \\
& =l+f^{T A L}\left(v(N)-\sum_{i \in N} l_{i}, u^{*}(v)-l\right) \\
& =l+f^{T A L}\left(v(N)-\sum_{i \in N} l_{i}, u-l\right) \\
& =u-f^{T A L}\left(\sum_{i \in N} u_{i}-v(N), u-l\right) .
\end{aligned}
$$


(iii) There exists $i \in N$ such that $l_{i}<l_{i}^{*}(v), u_{i}>u_{i}^{*}(v), l_{j}=l_{j}^{*}(v)$ and $u_{j}=u_{j}^{*}(v)$ for all $j \in N \backslash\{i\}$. By Theorem 2 .

$$
v(N)-\sum_{j \in N} l_{j}^{*}(v)=u_{i}^{*}(v)-l_{i}^{*}(v)=\sum_{j \in N} u_{j}^{*}(v)-v(N) .
$$

This implies that $v(N)=\frac{1}{2} \sum_{j \in N}\left(u_{j}^{*}(v)+l_{j}^{*}(v)\right)$. Then

$$
\begin{aligned}
\eta(v) & =l^{*}(v)+f^{T A L}\left(v(N)-\sum_{j \in N} l_{j}^{*}(v), u^{*}(v)-l^{*}(v)\right) \\
& =l^{*}(v)+f^{T A L}\left(\frac{1}{2} \sum_{j \in N}\left(u_{j}^{*}(v)-l_{j}^{*}(v)\right), u^{*}(v)-l^{*}(v)\right) \\
& =l^{*}(v)+\frac{1}{2}\left(u^{*}(v)-l^{*}(v)\right) \\
& =\frac{1}{2}\left(u^{*}(v)+l^{*}(v)\right) .
\end{aligned}
$$

Define $\left(E^{*}, c^{*}\right) \in \mathcal{B}^{N}$ by $E^{*}=v(N)-\sum_{j \in N} l_{j}^{*}(v)$ and $c^{*}=u^{*}(v)-l^{*}(v)$, and define $(E, c) \in \mathcal{B}^{N}$ by $E=v(N)-\sum_{j \in N} l_{j}$ and $c=u-l$. Then

$$
\begin{gathered}
E-E^{*}=\sum_{j \in N} l_{j}^{*}(v)-\sum_{j \in N} l_{j}=l_{i}^{*}(v)-l_{i}>0, \\
c_{i}-c_{i}^{*}=\left(u_{i}-u_{i}^{*}(v)\right)+\left(l_{i}^{*}(v)-l_{i}\right)>E-E^{*},
\end{gathered}
$$

and $c_{j}=c_{j}^{*}$ for all $j \in N \backslash\{i\}$. Moreover, for all $j \in N \backslash\{i\}$,

$$
c_{i}>c_{i}^{*}=u_{i}^{*}(v)-l_{i}^{*}(v)=v(N)-\sum_{k \in N} l_{k}^{*}(v) \geq u_{j}^{*}(v)-l_{j}^{*}(v)=c_{j}^{*}=c_{j} .
$$

This implies that $f_{i}^{T A L}(E, c)=f_{i}^{T A L}\left(E^{*}, c^{*}\right)+E-E^{*}=f_{i}^{T A L}\left(E^{*}, c^{*}\right)+l_{i}^{*}(v)-l_{i}$ and $f_{j}^{T A L}(E, c)=f_{j}^{T A L}\left(E^{*}, c^{*}\right)$ for all $j \in N \backslash\{i\}$. Applying Lemma 3 and self-duality,

$$
\begin{aligned}
\eta(v) & =l^{*}(v)+f^{T A L}\left(v(N)-\sum_{i \in N} l_{i}^{*}(v), u^{*}(v)-l^{*}(v)\right) \\
& =l^{*}(v)+f^{T A L}\left(E^{*}, c^{*}\right) \\
& =l+f^{T A L}(E, c) \\
& =l+f^{T A L}\left(v(N)-\sum_{i \in N} l_{i}, u-l\right) \\
& =u-f^{T A L}\left(\sum_{i \in N} u_{i}-v(N), u-l\right) .
\end{aligned}
$$




\section{Example 6}

Let $v \in \Gamma_{t}^{N}$ with $N=\{1,2,3\}$ be defined by $v(\{1\})=v(\{2\})=2, v(\{3\})=4, v(\{1,2\})=10$, $v(\{1,3\})=6, v(\{2,3\})=12$, and $v(N)=20$. Then $l^{*}(v)=(2,2,4)$ and $u^{*}(v)=(8,14,10)$. Since $l_{1}^{*}(v)+u_{2}^{*}(v)+l_{3}^{*}(v)=v(N)=u_{1}^{*}(v)+l_{2}^{*}(v)+u_{3}^{*}(v)$, Theorems 2 and 3 imply that

$$
\begin{aligned}
\eta(v) & =(2,2,4)+f^{T A L}(12,(6,12,6))=(8,14,10)-f^{T A L}(12,(6,12,6)) \\
& =(2,0,4)+f^{T A L}(14,(6,14,6))=(8,20,10)-f^{T A L}(18,(6,18,6)) \\
& =(2,0,4)+f^{T A L}(14,(6,20,6))=(8,20,10)-f^{T A L}(18,(6,20,6))=(5,8,7) .
\end{aligned}
$$

The first two expressions are in terms of the lower exact core bounds and the upper exact core bounds. The third expression is based on a decrease of only the lower exact core bound of player 2 to $l_{2}=0$. The fourth expression is based on an increase of only the upper exact core bound of player 2 to $u_{2}=20$. The fifth and sixth expressions are based on a decrease of player 2's lower bound to $l_{2}=0$ and an increase of player 2's upper bound to $u_{2}=20$ simultaneously. In view of $u_{1}^{*}(v)+l_{2}^{*}(v)+l_{3}^{*}(v)<v(N)<l_{1}^{*}(v)+u_{2}^{*}(v)+u_{3}^{*}(v)$ and $l_{1}^{*}(v)+l_{2}^{*}(v)+u_{3}^{*}(v)<v(N)<u_{1}^{*}(v)+u_{2}^{*}(v)+l_{3}^{*}(v)$, the lower and upper exact core bounds of players 1 and 3 cannot be stretched.

So far, we have studied to what extent the exact core bounds of a two-bound core game can be stretched while retaining the core and nucleolus descriptions. Instead of stretching the lower and upper bounds, we can also study to what extent these expressions are robust against game changes. It turns out that the worths of coalitions can be increased subject to specific restrictions.

\section{Theorem 4}

Let $v \in \Gamma_{t}^{N}$ and let $l, u \in \mathbb{R}^{N}$ be such that $C(v)=[l, u] \cap X(v)$. If $w \in \Gamma^{N}$ is such that $v(S) \leq w(S) \leq \max \left\{\sum_{i \in S} l_{i}, v(N)-\sum_{i \in N \backslash S} u_{i}\right\}$ for all $S \in 2^{N} \backslash\{\emptyset\}$, then the following statements hold:

(i) $C(v)=C(w)$,

(ii) $\eta(v)=\eta(w)$.

Proof. (i) Define $\hat{v} \in \Gamma^{N}$ by $\hat{v}(S)=\max \left\{\sum_{i \in S} l_{i}, v(N)-\sum_{i \in N \backslash S} u_{i}\right\}$ for all $S \in 2^{N} \backslash\{\emptyset\}$. By Theorem 1 $v(S) \leq \hat{v}(S)$ for all $S \in 2^{N} \backslash\{\emptyset\}$. Let $w \in \Gamma^{N}$ be such that $v(S) \leq w(S) \leq \hat{v}(S)$ for all $S \in 2^{N} \backslash\{\emptyset\}$. Then $C(\hat{v}) \subseteq C(w) \subseteq C(v)$. We claim that $C(\hat{v})=C(v)$. Suppose, to the contrary, that there exists $x \in C(v) \backslash C(\hat{v})$. Let $S \in 2^{N} \backslash\{N, \emptyset\}$ be such that $\sum_{i \in S} x_{i}<\hat{v}(S)$. If $\hat{v}(S)=\sum_{i \in S} l_{i}$, then $\sum_{i \in S} x_{i}<\sum_{i \in S} l_{i}$, so $x_{i}<l_{i}$ for some $i \in S$, contradicting $x \in C(v)$. If $\hat{v}(S)=v(N)-\sum_{i \in N \backslash S} u_{i}$, then $\sum_{i \in S} x_{i}<v(N)-\sum_{i \in N \backslash S} u_{i}$, so $x_{i}>u_{i}$ for some $i \in N \backslash S$, contradicting $x \in C(v)$. Hence, $C(v)=C(w)=C(\hat{v})$. 
(ii) Statement (i) implies that $w \in \Gamma_{t}^{N}$ and $C(w)=C(v)=[l, u] \cap X(v)=[l, u] \cap X(w)$. By Theorem 3 ,

$$
\eta(v)=l+f^{T A L}\left(v(N)-\sum_{i \in N} l_{i}, u-l\right)=l+f^{T A L}\left(w(N)-\sum_{i \in N} l_{i}, u-l\right)=\eta(w) .
$$

\section{Concluding remarks}

In this paper, we introduced the large class of two-bound core games and provided explicit expressions of the nucleolus in terms of all pairs of bounds describing the core, using the Talmud rule for bankruptcy problems. Other values for two-bound core games are directly obtained by replacing the role of the Talmud rule in these expressions by any other bankruptcy rule. Quant et al. (2006) studied these extensions from a general point of view and paid particular attention to the specific random arrival rule (cf. O’Neill 1982). González-Díaz et al. (2005) followed a similar approach with a focus on the adjusted proportional rule (cf. Curiel et al. 1987). Future research could study extensions of these and other bankruptcy rules to the class of two-bound core games. 


\section{Appendix}

\section{Proof of Theorem 1}

Proof. For the only-if part, assume that $C(v)=[l, u] \cap X(v)$. Then, according to Lemma 1 . we have $m^{l, u, \sigma}(v) \in C(v)$ for all $\sigma \in \Pi(N)$. Let $S \in 2^{N} \backslash\{\emptyset\}$ and consider $\sigma^{*} \in \Pi(N)$ such that $\sigma^{*}(k) \in N \backslash S$ for all $k \in\{1, \ldots,|N \backslash S|\}$. If the pivot player of $m^{l, u, \sigma^{*}}(v)$ is an element of $N \backslash S$, then $m_{i}^{l, u, \sigma^{*}}(v)=l_{i}$ for all $i \in S$, so

$$
v(S) \leq \sum_{i \in S} m_{i}^{l, u, \sigma^{*}}(v)=\sum_{i \in S} l_{i} .
$$

If the pivot player of $m^{l, u, \sigma^{*}}(v)$ is an element of $S$, then $m_{i}^{l, u, \sigma^{*}}(v)=u_{i}$ for all $i \in N \backslash S$, so

$$
v(S) \leq \sum_{i \in S} m_{i}^{l, u, \sigma^{*}}(v)=v(N)-\sum_{i \in N \backslash S} m_{i}^{l, u, \sigma^{*}}(v)=v(N)-\sum_{i \in N \backslash S} u_{i} .
$$

Combining these two cases, we obtain expression (1).

For the if-part, assume that expression (11) holds for all $S \in 2^{N} \backslash\{\emptyset\}$. We only need to prove that $[l, u] \cap X(v) \subseteq C(v)$. In view of the convexity of the core, together with Lemma 1. it suffices to show that $m^{l, u, \sigma}(v) \in C(v)$ for all $\sigma \in \Pi(N)$. For all $S \in 2^{N} \backslash\{\emptyset\}$ and all $\sigma \in \Pi(N)$

$$
\begin{aligned}
v(S) & \leq \max \left\{\sum_{i \in S} l_{i}, v(N)-\sum_{i \in N \backslash S} u_{i}\right\} \\
& \leq \max \left\{\sum_{i \in S} m_{i}^{l, u, \sigma}(v), v(N)-\sum_{i \in N \backslash S} m_{i}^{l, u, \sigma}(v)\right\}=\sum_{i \in S} m_{i}^{l, u, \sigma}(v) .
\end{aligned}
$$

Hence, $m^{l, u, \sigma}(v) \in C(v)$ for all $\sigma \in \Pi(N)$.

\section{Proof of Lemma 3}

Proof. Define $w \in \Gamma^{N}$ by $w(S)=v(S)-\sum_{i \in S} l_{i}^{*}(v)$ for all $S \in 2^{N}$. Then $l_{i}^{*}(w)=0$ and $u_{i}^{*}(w)=u_{i}^{*}(v)-l_{i}^{*}(v)$ for all $i \in N, C(w)=\left[l^{*}(w), u^{*}(w)\right] \cap X(w)$, and $\eta(v)=l^{*}(v)+\eta(w)$. For each $i \in N$, there exists $x \in C(w)$ such that $x_{i}=l_{i}^{*}(w)$, so

$$
0=l_{i}^{*}(w)=x_{i}=w(N)-\sum_{j \in N \backslash\{i\}} x_{i} \geq w(N)-\sum_{j \in N \backslash\{i\}} u_{j}^{*}(w) .
$$

Similarly, for each $i \in N$, there exists $x \in C(w)$ such that $x_{i}=u_{i}^{*}(w)$, so

$$
u_{i}^{*}(w)=x_{i}=w(N)-\sum_{j \in N \backslash\{i\}} x_{i} \leq w(N)-\sum_{j \in N \backslash\{i\}} l_{j}^{*}(w)=w(N) .
$$


Define $(E, c) \in \mathcal{B}^{N}$ by $E=w(N)$ and $c=u^{*}(w)$. Then $v_{E, c}(N)=E=w(N)$ and for all $i \in N$,

$$
l_{i}^{*}\left(v_{E, c}\right)=\max \left\{0, E-\sum_{j \in N \backslash\{i\}} c_{j}\right\}=\max \left\{0, w(N)-\sum_{j \in N \backslash\{i\}} u_{i}^{*}(w)\right\}=0=l_{i}^{*}(w)
$$

and

$$
u_{i}^{*}\left(v_{E, c}\right)=\min \left\{E, c_{i}\right\}=\min \left\{w(N), u_{i}^{*}(w)\right\}=u_{i}^{*}(w) .
$$

This implies that

$$
\begin{aligned}
C\left(v_{E, c}\right) & =\left[l^{*}\left(v_{E, c}\right), u^{*}\left(v_{E, c}\right)\right] \cap X\left(v_{E, c}\right) \\
& =\left\{x \in \mathbb{R}^{N} \mid \sum_{i \in N} x_{i}=v_{E, c}(N) \text { and } l^{*}\left(v_{E, c}\right) \leq x \leq u^{*}\left(v_{E, c}\right)\right\} \\
& =\left\{x \in \mathbb{R}^{N} \mid \sum_{i \in N} x_{i}=w(N) \text { and } l^{*}(w) \leq x \leq u^{*}(w)\right\} \\
& =\left[l^{*}(w), u^{*}(w)\right] \cap X(w) \\
& =C(w) .
\end{aligned}
$$

Potters and Tijs (1994) showed that the nucleoli of two balanced games are equal if their cores are equal and at least one of the two games is convex. Since $v_{E, c}$ is convex, this implies that $\eta\left(v_{E, c}\right)=\eta(w)$. Applying self-duality,

$$
\begin{aligned}
\eta(v) & =l^{*}(v)+\eta(w) \\
& =l^{*}(v)+\eta\left(v_{E, c}\right) \\
& =l^{*}(v)+f^{T A L}(E, c) \\
& =l^{*}(v)+f^{T A L}\left(w(N), u^{*}(w)\right) \\
& =l^{*}(v)+f^{T A L}\left(v(N)-\sum_{i \in N} l_{i}^{*}(v), u^{*}(v)-l^{*}(v)\right) \\
& =u^{*}(v)-f^{T A L}\left(\sum_{i \in N} u_{i}^{*}(v)-v(N), u^{*}(v)-l^{*}(v)\right) .
\end{aligned}
$$

\section{References}

Aumann, R. J. and M. Maschler (1985). Game theoretic analysis of a bankruptcy problem from the Talmud. Journal of Economic Theory, 36(2), 195-213. 
Bondareva, O. N. (1963). Some applications of linear programming methods to the theory of cooperative games. Problemy Kibernitiki, 10, 119-139.

Bondareva, O. N. and T. S. H. Driessen (1994). Extensive coverings and exact core bounds. Games and Economic Behavior, 6(2), 212-219.

Curiel, I. J., M. Maschler, and S. H. Tijs (1987). Bankruptcy games. Zeitschrift für Operations Research, 31(5), A143-A159.

Dietzenbacher, B. (2018). Bankruptcy games with nontransferable utility. Mathematical Social Sciences, 92, 16-21.

Driessen, T. S. H. (1986). $k$-convex $n$-person games and their cores. Zeitschrift für Operations Research, 30(1), A49-A64.

González-Díaz, J., P. Borm, R. Hendrickx, and M. Quant (2005). A geometric characterisation of the compromise value. Mathematical Methods of Operations Research, 61(3), $483-500$.

Muto, S., M. Nakayama, J. Potters, and S. H. Tijs (1988). On big boss games. The Economic Studies Quarterly, 39(4), 303-321.

O'Neill, B. (1982). A problem of rights arbitration from the Talmud. Mathematical Social Sciences, 2(4), 345-371.

Potters, J., R. Poos, S. H. Tijs, and S. Muto (1989). Clan games. Games and Economic Behaviour, 1(3), 275-293.

Potters, J. and S. H. Tijs (1994). On the locus of the nucleolus. In: N. Megiddo (Ed.), Essays in Game Theory: in honor of Michael Maschler, pp. 193-203. Berlin: SpringerVerlag.

Quant, M., P. Borm, R. Hendrickx, and P. Zwikker (2006). Compromise solutions based on bankruptcy. Mathematical Social Sciences, 51(3), 247-256.

Quant, M., P. Borm, H. Reijnierse, and B. V. Velzen (2005). The core cover in relation to the nucleolus and the Weber set. International Journal of Game Theory, 33(4), 491-503.

Schmeidler, D. (1969). The nucleolus of a characteristic function game. SIAM Journal on Applied Mathematics, 17(6), 1163-1170.

Shapley, L. S. (1967). On balanced sets and cores. Naval Research Logistics Quarterly, 14(4), 453-460.

Shapley, L. S. (1971). Cores of convex games. International Journal of Game Theory, 1, $11-26$.

Tijs, S. H. and F. A. S. Lipperts (1982). The hypercube and the core cover of $N$-person cooperative games. Cahiers du Centre d'Etudes de Recherche Opérationnelle, 24, 2737. 